\title{
Active Pedestrian Safety by Automatic Braking and Evasive Steering
}

\author{
C. Keller, T. Dang, H. Fritz, A. Joos, C. Rabe and D. M. Gavrila
}

\begin{abstract}
Active safety systems hold great potential to reduce the accident frequency and severity, by warning the driver and/or exerting automatic vehicle control ahead of crashes. This paper presents a novel active pedestrian safety system, which combines sensing, situation analysis, decision making and vehicle control. The sensing component is based on stereo vision; it fuses two complementary approaches for added robustness: motion-based object detection and pedestrian recognition. The highlight of the system is the ability to decide within a split second whether to perform automatic braking or evasive steering, and to execute this maneuver reliably, at relatively high vehicle speeds (up to $50 \mathrm{~km} / \mathrm{h}$ ).

We performed extensive pre-crash experiments with the system on the test track (22 scenarios with real pedestrians and a dummy). We obtained a significant benefit in detection performance and improved lateral velocity estimation by the fusion of motion-based object detection and pedestrian recognition. On a fully reproducible scenario subset, involving the dummy entering laterally into the vehicle path from behind an occlusion, the system executed in over 40 trials the intended vehicle action: automatic braking (if a full stop is still possible) or else, automatic evasive steering.
\end{abstract}

Index Terms - pedestrian detection, computer vision, active safety ITS, vehicle control

\section{INTRODUCTION}

Pedestrians are arguably the most vulnerable traffic participants; yearly fatality figures sum to about 5700 for the EU, 4700 for the USA, and 2300 for Japan [1]. These represent approximately $18 \%, 11 \%$ and $32 \%$ of all traffic fatalities in the respective regions. Nations with emerging economies have a higher pedestrian fatality incidence [1].

The past few years have seen increased awareness of the plight of vulnerable road users at the EU level. In 2003, the EU passed Phase 1 of Directive 2003/102/E on pedestrian protection, focussing on passive safety, i.e. meaning to reduce injury levels upon impact, by specifying various maximum impact criteria (e.g. head, leg). More recently, June 2008, the EU Parliament approved the Phase 2 draft legislation, which specifies a combination of passive and active safety measures. In particular, Phase 2 requires new passenger cars to be fitted with Brake Assist Systems (BAS) as early as 2009. Pedestrian protection is meanwhile also a major theme for consumer rating groups like Euro NCAP.

Passive pedestrian safety measures involve vehicle structures (e.g. bonnet, bumper) that expand during collision in order to minimize the impact of the pedestrian leg or head hitting the vehicle. For example, Mercedes-Benz introduced

C. Keller is with the University of Heidelberg, Germany

T. Dang, H. Fritz, A. Joos, C. Rabe and D. M. Gavrila are with Daimler R\&D, Germany

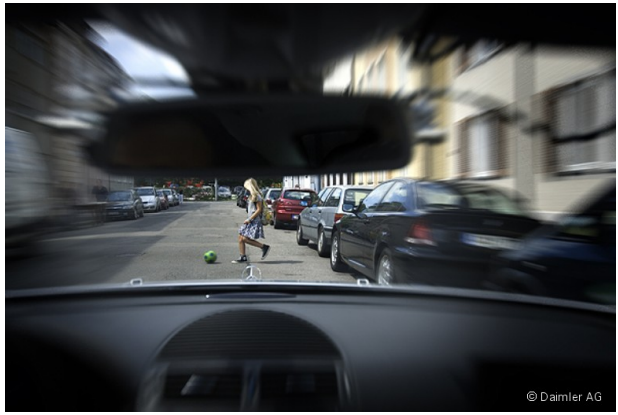

Fig. 1. Typical dangerous situation: a child running unexpectedly onto the street.

the active bonnet as standard for the new E-Class 2009. The system includes three impact sensors in the front section as well as special bonnet hinges pretensioned by powerful springs. Upon impact with a pedestrian, the rear section of the bonnet is pushed upwards by $50 \mathrm{~mm}$ in a fraction of a second, thus enlarging the deformation zone. The system is reversible and can be reset manually by the driver.

Although important, passive pedestrian safety measures are constrained by the laws of physics in terms of ability to reduce collision energy and thus injury level. Moreover, passive measures cannot account for injuries sustained in the secondary impact of the pedestrian hitting the road. Much effort is therefore spent towards the development of active driver assistance systems, which detect dangerous situations involving pedestrians ahead of time, allowing the possibility to warn the driver or to automatically control the vehicle. Such systems are particularly valuable when the driver is distracted or visibility is poor. See Figure 1.

The first night vision systems that detect and highlight pedestrians have reached the market (e.g. Mercedes-Benz EClass 2009 and BMW 7 series 2008). Volvo recently introduced in the S60 limousine a collision mitigation braking system for pedestrians, based on monocular vision and radar.

In this paper, we present a novel active pedestrian safety system, which combines sensing, situation analysis, decision making and vehicle control. The outline of the paper is as follows. Section II discusses previous work. The sensing component, based on stereo vision, consists of two complementary approaches, pedestrian recognition (Section III-A) and motionbased object segmentation (Section III-B), and their fusion (Section III-C). Situation analysis predicts how the current driving situation will evolve and automatically evaluates its criticality. This criticality assessment serves as the basis for a decision module which triggers appropriate maneuvres for collision avoidance and mitigation. Such maneuvers are realized by specialized vehicle controllers. Situation analysis, decision 
making and vehicle control are the topic of Section IV. We present the extensive experiments with the resulting system in pre-crash experiments on the test track in Section V. Future work and implications of such pre-crash safety systems are discussed in Section VI. We conclude in Section VII.

\section{Previous Work}

There exists meanwhile an extensive amount of literature on pedestrian safety. Gandhi and Trivedi [2] provide a broad survey on passive and active pedestrian protection methods, discussing multiple sensor types and methods for collision risk assessment. Enzweiler and Gavrila [3] focus in a more recent survey on techniques for video-based pedestrian detection. A large dataset (8.5GB) with many tens of thousands of labeled pedestrians was made public for benchmarking purposes.

One can decompose video-based pedestrian detection systems roughly into three components: the generation of initial object hypotheses (ROI selection), verification (classification), and temporal integration (tracking). We only provide a brief discussion, for a more complete discussion, see the survey article [3].

The simplest way to obtain ROIs is by means of a sliding window approach, where detector windows at various scales and locations are shifted over the image. Significant speedups can be obtained by coupling the sliding window approach with a classifier cascade of increasing complexity [4], [5] or by restricting the search space given known camera geometry and certain assumptions (i.e. flat-world, pedestrians on groundplane, typical pedestrian sizes). Other ROI selection techniques use stereo vision [6], [7], [8], [9], [10] or motion cues [11].

Pedestrian classification can be performed using either generative or discriminative models. Generative approaches model pedestrian appearance in terms of its class-conditional density function. In combination with the class priors, the posterior probability for the pedestrian class can be inferred using a Bayesian approach. Most generative approaches use shape [9], [12] or combined shape-texure cues [13]. Discriminative models approximate the Bayesian maximum-a-posteriori decision by learning the parameters of a discriminant function (decision boundary) between the pedestrian and nonpedestrian classes from training examples. Among the more popular image features used in this context are Haar wavelets [14], code-book feature patches [8], histograms of oriented gradients (HOG) [15], and local receptive fields [9]. There is a recent trend towards classifier ensembles [16] or mixture-of-experts [17] for improved performance.

Regarding tracking, one line of research has considered this as frame-by-frame association of detections based on geometry and dynamics without particular pedestrian appearance models [6], [9]. Other approaches utilize pedestrian appearance models coupled with geometry and dynamics [8], [10]. Some approaches (e.g. [10]) furthermore integrate detection and tracking in a Bayesian framework, combining appearance models with an observation density, dynamics, and probabilistic inference of the posterior state density.

A number of pedestrian systems were installed on-board vehicles [18], [19], [20], [9], [21], [22], [23], [24]. Some of these not only implement a perception component but also collision risk estimation in combination with acoustical driver warning and/or automatic vehicle braking, see systems by Daimler [22], Ibeo [20], VW [22], [23], and the Universities of Alcala [21] and Parma [19]. Other work dealt with pedestrian perception, collision risk estimation and vehicle actuation by means of simulation [25].

Systems for collision avoidance and mitigation by braking are already in the market for passenger cars and commercial vehicles and suitable methods for criticality assessment have already been been proposed (e.g. [26]). However, collision avoidance by steering has not been covered in depth in the literature. Most work on trajectory generation for collision avoidance has been done in the robotics field. Powerful methods to solve non-holonomic motion planning problems with dynamic obstacles have been proposed (e.g. [27], [28]), yet the computational complexity of many of the proposed algorithms prohibits the application on current automotive hardware. To overcome this limitation, efficient planning algorithms to evaluate possible collision avoidance maneuvers by human drivers in highly structured scenarios have been introduced [29]. Optimal vehicle trajectory control for obstacle avoidance within shortest distance is presented in [30]. The Proreta Project [31] evaluated driver assistance systems that initiate automatic braking when an object vehicle cuts into the ego vehicle's lane and automatic steering when an object vehicle is standing in front of the ego vehicle and the driver does not react. Other systems that performed automated steering have been demonstrated at the DARPA Urban Challenge [32]. However, the latter systems mostly used expensive sensors that are not suited for the automotive context (e.g. Velodyne scanners) and executed steering maneuvers at relatively low vehicle speeds.

The contributions of this paper are as follows. The main contribution is the description of an integrated active pedestrian safety system, which combines sensing, situation analysis, decision making and vehicle control. The secondary contribution concerns the sensing component; it is based on stereo vision and fuses two complementary approaches for added robustness: motion-based object segmentation and pedestrian recognition. The highlight of the system is the ability to decide within a split second whether to perform automatic braking or evasive steering, and to execute this maneuver reliably, at relatively high vehicle speed (up to $50 \mathrm{~km} / \mathrm{h}$ ).

\section{Video-BAsed Pedestrian SEnsing}

\section{A. Single-Frame Pedestrian Recognition (PedRec)}

Initial regions of interest (ROIs) are generated using the sliding window technique described in [9]. The depth image, obtained by stereo vision, is scanned with windows related to the maximum extents of pedestrians, assuming the latter are standing on the ground plane, while taking into account appropriate positional tolerances (e.g. vehicle pitch, slightly curved roads vertically). The locations where the number of (depth) features exceeds a percentage of the window area are added to the ROI list for the subsequent pedestrian classification. Candidates are classified following the HOG/linSVM approach of Dalal and Triggs [15]. Multiple detector responses 
at near identical locations and scales are addressed by applying confidence-based non-maximum suppression to the detected bounding boxes using pairwise box coverage: two system detections $a_{i}$ and $a_{j}$ are subject to non-maximum suppression if their coverage $\Gamma\left(a_{i}, a_{j}\right)=\frac{A\left(a_{i} \cap a_{j}\right)}{A\left(a_{i} \cup a_{j}\right)}$, the ratio of intersection area and union area, is above $\theta_{n}$.

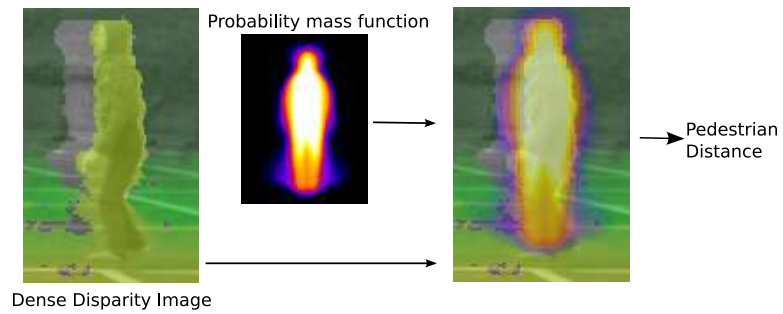

Fig. 2. Estimating pedestrian distance from dense stereo using a probability mass function derived from manually labeled pedestrian shapes. By averaging the weighted distance values the pedestrian distance can be computed.

The distance of a detected pedestrian in the image is estimated using the computed dense stereo image. Because the exact contour of the pedestrian is unknown all possible pedestrian shapes are considered in the depth estimation process using a probability mass function, as described in [11]. Figure 2 illustrates the depth estimation procedure. Distance values in the depth image for a given bounding box are weighted and averaged using the probability mass function. The 3D position of the pedestrian is given by backprojecting the vertical line going through the bounding box center and the computed box distance. Detected 3D pedestrian locations are passed untracked to the fusion module.

\section{B. Detection of Moving Objects (6D-Vision)}

Using a stereo camera set-up, the 3D structure of the observed scene can be immediately obtained by a stereo algorithm (e.g. [33], [34]). Usually, to identify individual objects, this information is accumulated in an evidence-grid-like structure, followed by a connected-component analysis [35]. To obtain the motion of the identified objects, the objects are then tracked over time and their velocity is estimated by means of filtering. The disadvantage of this standard approach is that the performance of the detection depends highly on the correctness of the segmentation. Especially moving objects close to stationary ones - e.g. the moving pedestrian behind the standing vehicle are often merged and therefore not detected.

To overcome this problem, we proposed in [36], [37] to base the detection not only on the stereo information, but also on the 3D motion field. The reconstruction of the 3D motion field is performed by the so called 6D-Vision algorithm. The basic idea is to track points with depth known from stereo vision over two and more consecutive frames and to fuse the spatial and temporal information using Kalman filters. The result is an improved accuracy of the 3D-position and an estimation of the 3D-motion of the considered point at the same time. This fusion implies the knowledge of the motion of the observer, also called the ego-motion. It is estimated from the image points found to be stationary, using a Kalman filter based approach. However, other methods, like for example [38] or [39] can be easily integrated.

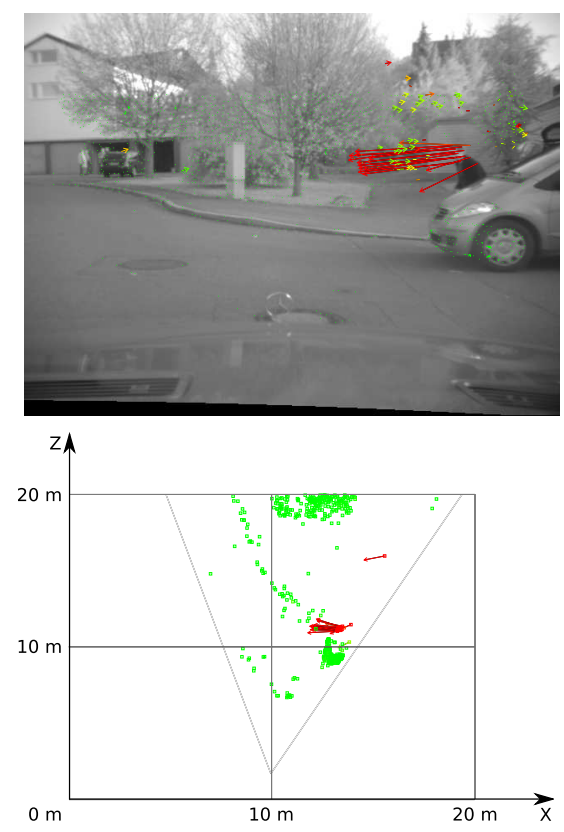

Fig. 3. Estimation result of the $6 \mathrm{D}$-Vision algorithm. The arrows point to the estimated 3D position in $0.5 \mathrm{~s}$, projected back onto the image. The color encodes the absolute velocity: Static points are encoded green, points moving at a speed of $4.0 \mathrm{~m} / \mathrm{s}$ or above are encoded red.

In the current setup, the image points are tracked by a KLT tracker [40], which provides sub-pixel accuracy and tracks the image points robustly for a long sequence of images. It was optimized with respect to speed, allowing the complete motion-based object detection module to analyze up to 5000 points in real-time ( $25 \mathrm{fps})$. The stereo computation is performed by a hardware implementation of the semi-global matching algorithm [34]. However, any comparable optical flow and stereo algorithms can be used.

The estimation result of the $6 \mathrm{D}$-Vision algorithm is shown in Figure 3. Here, the arrows point from the current 3D-position to the predicted 3D-position in $0.5 \mathrm{~s}$. Looking at the bird's-eye view in the right image, the moving pedestrian is now easily distinguished from the standing vehicle.

Objects are identified as groups of contiguous coherent motion vectors. Since the $6 \mathrm{D}$-Vision algorithm provides not only the state estimates, but also their uncertainty, the Mahalanobis distance is used as a similarity measure in the cluster analysis.

\section{Fusion of Motion-based Object Detection (6D-Vision) and Pedestrian Recognition (PedRec)}

For an accurate prediction of pedestrian movement, both positional and velocity information is important. Input from 6D-Vision and PedRec modules are fused using a Kalman filter. The state $\mathbf{S}$ of the filter is modeled as

$$
\mathbf{S}=\left[\begin{array}{llll}
x & y & v_{x} & v_{y}
\end{array}\right]^{T}
$$

with $x / y$ being the longitudinal/lateral position of the pedestrian to the vehicle and $v_{x} / v_{y}$ being its absolute longitudinal/lateral velocity in the world. The measurement vectors associated with the 6D-Vision and PedRec modules are

$$
\mathbf{z}_{6 d}=\left[\begin{array}{llll}
x & y & v_{x} & v_{y}
\end{array}\right]^{T}, \quad \mathbf{z}_{p e d}=\left[\begin{array}{ll}
x & y
\end{array}\right]^{T},
$$

where $x / y$ and $v_{x} / v_{y}$ are various measurements of the state variables defined above (the mapping from state to mea- 
surements is thus trivial). Current measurements from both modules are integrated into the filter using successive update steps.

We assume a constant velocity pedestrian motion model (acceleration is modeled in the process noise covariance). The transition matrix $F$ is given by

$$
\mathbf{F}=\left(\begin{array}{cccc}
1 & 0 & \mathrm{~T} & 0 \\
0 & 1 & 0 & \mathrm{~T} \\
0 & 0 & 1 & 0 \\
0 & 0 & 0 & 1
\end{array}\right)
$$

with $T$ being the cycle time of the camera (40 ms).

Ego-motion of the vehicle is compensated in the prediction step of the Kalman Filter. Object translation with respect to the vehicle can be computed assuming a "bicycle" model [41] for the vehicle motion with constant steering angle and velocity between two measurement points. The required velocity and yaw rate data for the ego-motion-compensation is given by on-board sensor data and is accessible in the camera cycle time.

Measurement to track association is done using a global nearest neighbor (GNN) approach with prior rectangular gating on object positions. The Mahalanobis distance between predicted state and measurement is used for the data association. For pedestrian detections this means the position is used for measurement to track association, while for $6 \mathrm{D}$-Vision detections the velocity is used additionally.

Track initialization and termination is handled depending on the number of associations to a track. New tracks are initialized using measurements that could not be assigned to an existing track. In order to suppress spurious detections, tracks start in the state hidden. A track enters the state confirmed after a certain number $n$ of measurements have been assigned to the track. Here we use $n=2$, which means a detection from both modules at the same time directly results in a confirmed pedestrian track. Only tracks where a pedestrian detection has been assigned to are marked as valid pedestrian track. For all tracks a history of their state over time, including measurement to track associations is kept. Tracks are terminated after a user defined number of missed associations $m$.

Both modules operate independently at different cycle times. The 6D-Vision module operates in the fixed camera cycle time ( $25 \mathrm{fps})$. Processing time of the PedRec module varies depending on the scene complexity with a lower limit of 15 fps. Measurements have a common time-stamp defined by the frame-stamp of the image they have been generated on. In situations where measurements arrive out of sequence and can not be integrated in the common filter state, the track history is used to check measurements to track associations in the past. Possible assignments lead to an update of the association information. Although the filter state is not updated using the out of sequence measurements the updated association information effects the track management, allowing a track to enter the state confirmed. Additionally PedRec associations lead to a validated pedestrian track.

The initial state of the Kalman filter is derived from the first measurement. If a track is initialized by a pedestrian detection the velocities of the system state are set to zero. A track started

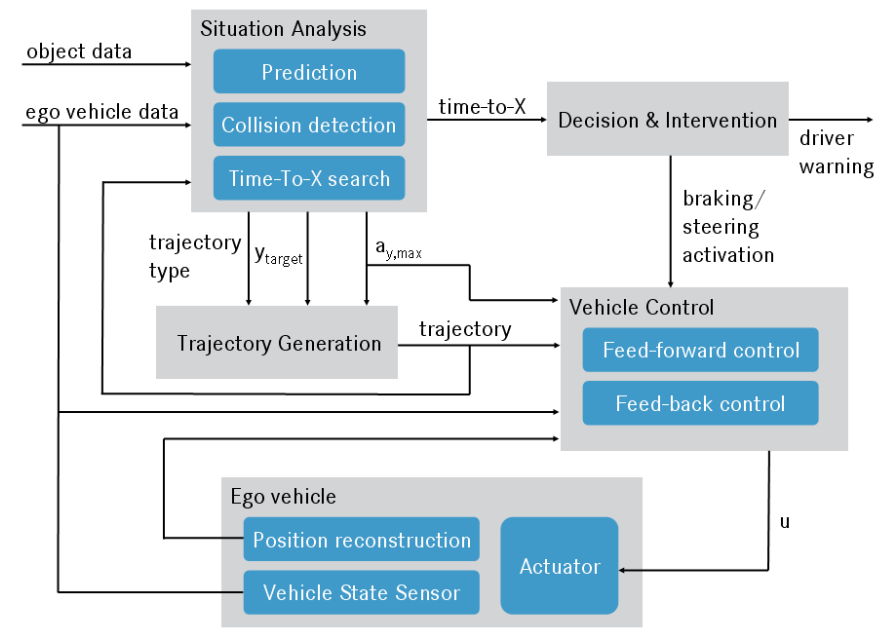

Fig. 4. System structure of situation analysis and vehicle control.

by a $6 \mathrm{D}-$ Vision detection uses the measured velocities as initial value.

Finally, position, velocity and extent of the tracked pedestrians are passed to the situation analysis module.

\section{Situation Analysis, Decision, Intervention, AND VEHICLE CONTROL}

Situation analysis and vehicle control are the components of a driver assistance system which generate a machine level understanding of the current situation (based on the previously described sensor information) and take appropriate actions. Figure 4 depicts the relationships between trajectory generation, situation analysis, decision \& intervention, and vehicle control.

Situation analysis predicts how the current driving situation will evolve and automatically evaluates its criticality using measures as e.g. time-to-collision, time-to-steer, and time-tobrake. This criticality assessment serves as the basis for a decision module which triggers appropriate maneuvers for collision avoidance and collision mitigation. Such maneuvers are realized by specialized vehicle controllers. Naturally, vehicle control and situation analysis are closely coupled, since both rely on accurate, realistic models of evasive maneuvers. These models are provided by a trajectory generation module. The following sections will discuss the aforementioned modules in more detail.

\section{A. Trajectory Generation}

The objective of trajectory generation is twofold. First, trajectory generation has to provide accurate models of evasive steering maneuvers that fulfill several requirements: The generated trajectory for evasion should be as comfortable as possible, feasible (i.e. drivable by the ego vehicle), and should also lead to a safe transition with minimal side-slipping of the vehicle during the automatic evasive maneuver. Snatch of steering wheel can be dangerous and must therefore be avoided.

Second, trajectory generation should also provide the reference input variables for lateral control such as yaw angle, yaw rate, etc. Different trajectory types have been investigated and a sigmoidal blending function based on a polynomial approach 
as proposed in [42] is used to model the evasive maneuver path.

A polynomial model of seventh degree for the evasive path

$$
y_{t r j}=f(x)=\sum_{i=0}^{7} b_{i} \cdot x^{i}
$$

where $y_{t r j}$ is the desired lateral and $x$ the longitudinal offset from the starting point of the evasion maneuver, allows to fulfill the requirements regarding comfort and feasibility. To meet these specifications, the determination of the polynomial coefficients $b_{i}$ is based on several constraint equations which limit the maximum lateral acceleration $a_{y, \max }$, the derivatives of the lateral offset and of the curvature, respectively.

Let $D_{e v}$ denote the required distance to complete evasive maneuver and $y_{\text {target }}$ the target lateral offset at the end of the maneuver. For the derivation of the polynomial coefficients $b_{i}$ in Eq. (2), we impose the following boundary conditions:

$$
\begin{array}{ll}
f(0)=0, & f\left(D_{e v}\right)=y_{\text {target }}, \\
\frac{d}{d x} f(0)=0, & \frac{d}{d x} f\left(D_{e v}\right)=0, \\
\frac{d^{2}}{d x^{2}} f(0)=0, & \frac{d^{2}}{d x^{2}} f\left(D_{e v}\right)=0 \\
\frac{d^{3}}{d x^{3}} f(0)=0, & \frac{d^{3}}{d x^{3}} f\left(D_{e v}\right)=0 .
\end{array}
$$

To ensure that the evasive path $y_{t r j}$ meets our requirements regarding comfort and feasibility, we require that the lateral acceleration of the vehicle stays within predefined bounds:

$$
\left|\frac{d^{2} f}{d t^{2}}\right|=\frac{d}{d t}\left\{\frac{d f}{d x} \frac{d x}{d t}\right\}=\frac{d^{2} f}{d x^{2}}\left(\frac{d x}{d t}\right)^{2}+\frac{d f}{d x} \frac{d^{2} x}{d t^{2}} \leq a_{y, \max } .
$$

With the simplifying assumption that the vehicle's motion in $x$-direction is constant during the evasive maneuver, i.e. $\frac{d x}{d t}=$ $v, \frac{d^{2} x}{d t^{2}}=0$, we can transform the inequality (7) to an extremal value problem,

$$
\left.v^{2} \frac{d^{2} f}{d x^{2}}\right|_{\tilde{x}}=a_{y, \max }
$$

where $\tilde{x}$ is the position of the extremum, i.e.

$$
\left.\frac{d^{3} f}{d x^{3}}\right|_{\tilde{x}}=0 \text {. }
$$

Eqs. (3-6) and (8-9) provide a set of ten equations sufficient to derive the eight polynomial coefficients $b_{0}, \ldots, b_{7}$, the maneuver length $D_{e v}$, and the position of the extremum $\tilde{x}$.

The transition time for evasion $T_{e v}$ can be approximated using the simplifying assumption of constant vehicle speed $v$

$$
T_{e v}=\frac{D_{e v}}{v}=K \cdot \sqrt{y_{\text {target }} / a_{y, \max }}
$$

where $K \approx 2.741$ is a constant shape factor for the polynomial of seventh degree that can be computed from the equations defined above.

Based on the polynomial function and on the measured vehicle velocity $v$, the important input variables (lateral offset $y_{t r j}$, curvature $c_{t r j}$, heading angle $\chi_{t r j}$ ) are determined for lateral control at every sample time step, see Figure 5.

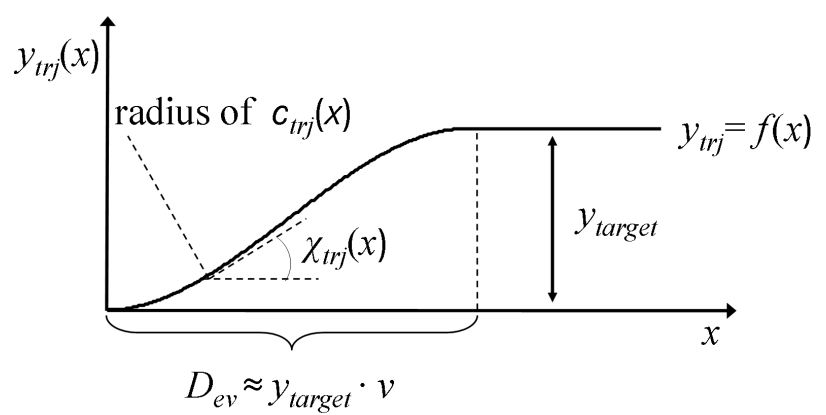

Fig. 5. Lateral control inputs from evasive path trajectory.

\section{B. Situation Analysis}

A commonly employed approach for collision risk assessment involves criticality measures such as Time-To-Brake (TTB), Time-To-Steer (TTS), etc.. TTB, for example, denotes the remaining time span in which the driver can still avoid a collision by braking with maximum deceleration. Detailed descriptions of Time-To-X criticality measures and their application in driver assistance systems for collision avoidance and mitigation can be found in [26].

In this paper, TTB and TTS are used to trigger automatic collision avoidance by either braking or steering maneuvers. There are, however, two important differences to standard Time-To-X computation: First, an evasive steering maneuver is commonly modeled as driving on a circular path with maximum lateral acceleration $a_{y, \max }$. Here, we employ a more realistic steering maneuver as defined in Section IV-A. Second, if TTS is used to assess the system's ability to avoid a collision by steering, we have to consider more than one relevant object. This means that the algorithm not only needs to find the latest steering maneuver which avoids a collision with the pedestrian in our driving path, but also has to ensure that the emergency maneuver does not result in a collision with any other detected object in the scene (e.g. cars, pedestrians; the integration of such free-space sensing component is left for future work, see Section VI). To fulfill these requirements we employ a numerical simulation method, which allows efficient, real time computation of Time-To-X criticality measures even for complex maneuvers. In addition, this numerical method can verify if an evasive steering maneuver can be performed without collision.

As depicted in Figure 4, the numerical simulation methods consist of three main components: prediction, collision detection, and Time-To-X search. In the prediction step, a sequence of potential future ego and other object states

$$
\left\{t_{k}, \mathbf{z}_{e g o, k}, \mathbf{z}_{o b j, k}^{1}, \ldots, \mathbf{z}_{o b j, k}^{M}\right\}, \quad k=1 \ldots K,
$$

is computed, where $t_{k}$ is the $k$-th time stamp of the prediction, $K$ the prediction horizon, $\mathbf{z}_{e g o, k}$ a vector describing the ego vehicle's pose and motion at time $t_{k}$, and $\mathbf{z}_{o b j, k}^{1}, \ldots, \mathbf{z}_{o b j, k}^{M}$ the pose and motion of all $M$ objects provided by the sensor data fusion (Section III-C). To obtain these predictions, we rely on appropriate motion models for all objects and the ego vehicle, thus making assumptions on their future behaviors.

Given the predicted states, we can identify potential collisions between the system vehicle and all objects in the scene by intersecting corresponding positions resulting from $\mathbf{z}_{e g o, k}$ 
and $\mathbf{z}_{o b j, k}^{1}, \ldots, \mathbf{z}_{o b j, k}^{M}$, respectively. If a collision is detected, we start the search for the latest possible collision avoidance maneuver.

To accomplish this task, we have defined two emergency maneuvers representing braking with maximum deceleration of $-10 \mathrm{~m} / \mathrm{s}^{2}$ and steering as modeled in Section IV-A, respectively. Each pairing $\left(t_{k}, \mathbf{z}_{e g o, k}\right)$ of Eq. (11) constitutes a potential starting point for an automatic emergency maneuver. Using a binary search algorithm, we can efficiently find the latest time steps at which braking or steering maneuvers have to be triggered that do not lead to a collision with any object in the scene. These time steps are discrete estimates of TTB and TTS.

\section{Decision \& Intervention}

The "decision \& intervention" is the core module of the assistance system, since it associates the function with the driver's behavior. Due to the high injury risk of a pedestrian in an accident, collision avoidance is the primary objective of the function. In order to identify the best way to support the driver, it is necessary to know the driver's current driving intention. The driver monitoring algorithm is using signals from the vehicle, e.g. accelerator and brake pedal position, speed, lateral and longitudinal acceleration, steering angle and steering rate to determine the current driving maneuver of the driver. If the driver is not reacting appropriately to the dangerous situation, an optical and acoustic warning will be given, so he can avoid the collision himself. In the case a function intervention is necessary to avoid the collision, full braking takes priority over the evasive maneuver. The full braking will be triggered when $T T B=0$ and the driver is neither doing an accelerating nor an evasive maneuver. If the collision cannot be prevented with full braking any more $(T T B<0)$, the evasive steering maneuver will be activated at $T T S=0$, provided the situation analysis has computed that this can be executed without collision; the evasive maneuver using the vehicle control to compute the necessary steering torque. The function ramps down the steering torque, when the evasive maneuver has finished. Afterwards the function is available immediately, when needed. Automatic evasion results in a fixed lateral offset of the vehicle in the range of $80-100 \mathrm{~cm}$. In case collision free evasive steering would not be possible because of, say, detected oncoming traffic, the decision would be to brake (collision mitigation).

The design of the prototype function allows the driver to overrule the steering intervention at any time. If the driver holds the steering wheel, he will weaken or suppress the steering of the system. A distinct activity of the accelerator or brake pedal cancels the evasive maneuver immediately. Similar exit conditions exist for the full braking intervention.

In order to minimize dynamic misalignments of the passengers during the system intervention additional protective measures are triggered. The function controls the electromotive reversible seatbelt pretensioners and the side-gated air cushions of the seating and backrests will be inflated. When the system has finished the intervention, the tension of the reversible seat belt pretensioners is released automatically and the air cushions of the seats are vented to the previous position.

\section{Vehicle Control}

Vehicle control consists of two parts: longitudinal control for automatic braking and lateral control for evasion. Automatic braking is triggered when $\mathrm{TTB}=0$ s (i.e. at the latest point in time when the ego vehicle can avoid the collision by full emergency braking), thus the longitudinal vehicle controller will set a maximum decceleration of $-10 \mathrm{~m} / \mathrm{s}^{2}$. The lateral control for evasive steering, however, is more complicated and will be discussed in the following sections.

Steering maneuvers for automatic collision avoidance entail highly dynamic lateral movements of the ego vehicle (here, lateral motion refers to motion perpendicular to our driving lane). The dynamics of such maneuvers with high lateral acceleration are nonlinear. In general, the lateral offset $y_{\text {target }}$ as defined in Section IV-A may vary from only a few centimeters to a full lane change depending on the size of the obstacle, its velocity, and the free space available for the evasive maneuver. Here, however, for pedestrian evasion a fixed lateral offset is used.

Collision avoidance by steering requires precise lateral control of the ego vehicle. The controller permanently compares the reference position along the evasive maneuver trajectory as specified in Eq. (2) to the actual vehicle position and thus requires accurate and reliable knowledge of the ego vehicle's pose.

The position of the vehicle is reconstructed from odometers and inertial sensors readily available in today's vehicles. Using the measured lateral acceleration $a_{y}$ and the velocity $v$ (or the current yaw rate $\dot{\psi}$, alternatively), the vehicle's heading angle $\chi$ can be recovered following

$$
\chi\left(t_{k}\right)=\chi\left(t_{k}-\Delta T\right)+\frac{a_{y}\left(t_{k}\right)}{v\left(t_{k}\right)} \Delta T
$$

or

$$
\chi\left(t_{k}\right)=\chi\left(t_{k}-\Delta T\right)+\dot{\psi}\left(t_{k}\right) \Delta T,
$$

respectively. Here, $\Delta T$ denotes the sampling time step and $t_{k}$ specifies the time stamp of the $k$-th iteration step. Using $\chi$ and the measured velocity $v$, numerical integration yields the longitudinal position $x$ and the lateral position $y$ with respect to the current lane

$$
\left(\begin{array}{l}
x\left(t_{k}\right) \\
y\left(t_{k}\right)
\end{array}\right)=\left(\begin{array}{c}
x\left(t_{k}-\Delta T\right) \\
y\left(t_{k}-\Delta T\right)
\end{array}\right)+v\left(t_{k}\right) \Delta T\left(\begin{array}{c}
\cos \chi\left(t_{k}\right) \\
\sin \chi\left(t_{k}\right)
\end{array}\right) .
$$

To account for the nonlinear lateral dynamics of the evasive maneuver, a control strategy combining feed forward and feed back control is used, i.e. the command signal $u$ of the lateral controller comprises the components $u_{f f}$ from a feed forward and $u_{f b}$ from feed back controller, respectively. $u_{f f}$ is computed from the trajectory curvature $c_{t r j}$ that can be derived from the polynomial in Eq. (2). The feed back component $u_{f b}$ is provided by a $4^{t h}$ order state controller with state vector $\left(y_{e r r}, \dot{y}_{e r r}, \chi_{e r r}, \dot{\chi}_{e r r}\right)$. Here, $y_{e r r}=y_{t r j}-y$ denotes the lateral position error between the reference lateral position and the reconstructed position, $\chi_{e r r}=\chi_{t r j}-\chi$ the difference between reference and reconstructed heading angle. $\dot{y}_{e r r}$ and $\dot{\chi}_{e r r}$ represent temporal derivatives which can be computed using either derivative lag (DT1) elements, state variable filters, or state observers. 
Due to the nonlinear behaviour of the vehicle, a gain scheduling approach is employed which adapts both the feed forward gain factor $K_{f f}$ and the feed back gain vector $\mathbf{K}_{f b}$ to the current velocity and the maximum allowed lateral acceleration $a_{y, \max }$, i.e. $K_{f f}=f\left(a_{y, \max }, v\right)$ and $\mathbf{K}_{f b}=$ $f\left(a_{y, \max }, v\right)$. Detailed information can be found in [43].

\section{EXPERIMENTS}

\section{A. Set-up}

Our vehicle prototype is a Mercedes-Benz S-class, with a stereo camera mounted behind the windshield. The stereo base line is $30 \mathrm{~cm}$ and each camera has a resolution of $640 \times$ 480 pixels and a focal length of $12 \mathrm{~mm}$. Two computers are mounted in the trunk; a $4 \mathrm{GHz}$ Quad Core Pentium with the image processing and fusion algorithms and a $2.2 \mathrm{GHz}$ dual core Pentium with the function specific algorithms. They are connected to the CAN network of the vehicle, which provides the required vehicle signals, such as speed and steering angle.

The vehicle prototype works with a conventional power steering, as it is used in the production vehicles. In addition, the steering possesses an electric steering torque actuator. It allows inducing an additional steering torque up to 5 $\mathrm{Nm}$ at the steering wheel to realize the automatic evasive maneuver. Braking and driver warning (display instrument panel, loudspeakers) were implemented using the MercedesBenz series control units. In addition, seat air cushions were inflated and seat belts were pre-tensioned in the event of a near-crash.

In order to test the prototype functionality, a traverse construction was installed on a proving ground, under which a pedestrian dummy, hung by a set of wires, can be moved across the road. An electronic device allowed reproducible movement of the pedestrian dummy. The synchronisation of the pedestrian dummy and the vehicle was achieved by a light barrier.

\section{B. Test of Video Sensing Component}

We first discuss the evaluation of the video sensing component only. A total of 22 scenarios were staged, covering real world situations of varying complexity, see Figure 6. The scenarios involve different numbers of pedestrians, geometrical lay-outs, walking speeds and visibility conditions. For safety reasons, lateral pedestrian movement resulting in nearcollisions was solely performed with the dummy. Furthermore, vehicle speed was reduced to $30 \mathrm{~km} / \mathrm{h}$ in those scenarios ( $\mathrm{S} 11$, $\mathrm{S} 13, \mathrm{~S} 17, \mathrm{~S} 21, \mathrm{~S} 22)$ where a real pedestrian was nearing the vehicle side up to $1.5 \mathrm{~m}$.

$3 \mathrm{D}$ ground truth positions of pedestrians with respect to the vehicle were obtained by manual labeling the corresponding bounding boxes in the camera images and by triangulation. Partially occluded pedestrians were labeled by a bounding box containing the visible body parts. We defined a sensor coverage range of 7-27 $\mathrm{m}$ in front and up to $6 \mathrm{~m}$ to each side of the vehicle medial axis, which was covered by both the 6DVision and PedRec modules. In this area all pedestrians were 'required', i.e. were needed to be detected by the system (even if only partially visible). Outside this area, pedestrians were 'optional', we did not credit or penalize for system detections.
In all, this resulted in 48 required pedestrian trajectories and 1700 pedestrian single-frame instances. We now consider four performance metrics in turn: detection performance, positionand speed-accuracy and time-to-detect.

Detection performance is related to the number of matches between ground truth and system-detected object locations. There are two aspects: sensitivity and precision. Sensitivity relates to the percentage of true solutions that were found by the system (i.e. detection percentage), whereas precision relates to the percentage of system solutions that were correct. A sensitivity and precision of $100 \%$ is ideal: the system finds all real solutions and produces no false positives. For additional insight, we consider the two criteria on both the frame- and trajectory-level. For the latter, we distinguish three types of trajectories: "class-a", "class-b", "class-c", which have $75 \%, 50 \%$ and 1 frame entries matched. Thus, all "classa" trajectories are also "class-b" trajectories, all "class-b" trajectories are also "class-c" trajectories; the three classes of trajectories represent different quality levels that might be relevant for particular applications.

In comparing system output with ground truth, we need to specify the localization tolerance, i.e. the maximum positional deviation that still allows us to count the system detection as a match. This localization tolerance is the sum of an applicationspecific component (how precise does the object localization have to be for the application) and a component related to measurement error (how exact can we determine true object location). We define object localization tolerance as percentage of distance, for longitudinal and lateral direction ( $X$ and $Y$ ), with respect to the vehicle. For our evaluation of the video sensing component, we took $X=15 \%$ and $Y=4 \%$, which means that, for example at $10 \mathrm{~m}$ distance, we tolerate a localization error (including ground truth measurement error) of \pm 1.5 and $\pm 0.4 \mathrm{~m}$ in the position of the pedestrian, longitudinal and lateral to the vehicle driving direction, respectively.

For this application we allow many-to-many correspondences. A ground truth location is considered matched if there is at least one system detection matching it. In practice, this means that in the case a group of pedestrians walking sufficiently close together in front of the vehicle, the system would not necessarily have to detect all of them in isolation, it suffices if each true pedestrian is within the localization tolerance of a detected pedestrian.

Table I summarizes the pedestrian detection performance. First two columns relate to 6D-Vision and (single-frame) PedRec output, which form the components of the fused system, shown in the last column. The third column represents the baseline case (termed 'PedRecTrack'): PedRec in combination with the previously described Kalman filter, without integrating the 6D-Vision detections. Two consecutive detections are required for a track to be initialized. After three missed detections tracks are closed.

From Table I one observes an improved performance of the fusion system (fourth column) vs. the baseline PedRec tracking system (third column). This is mainly due by the successful detections of 6D-Vision of the partially occluded pedestrians (i.e. upper body visible above parked car), which are not captured by the current PedRec, see Figure 9. By 

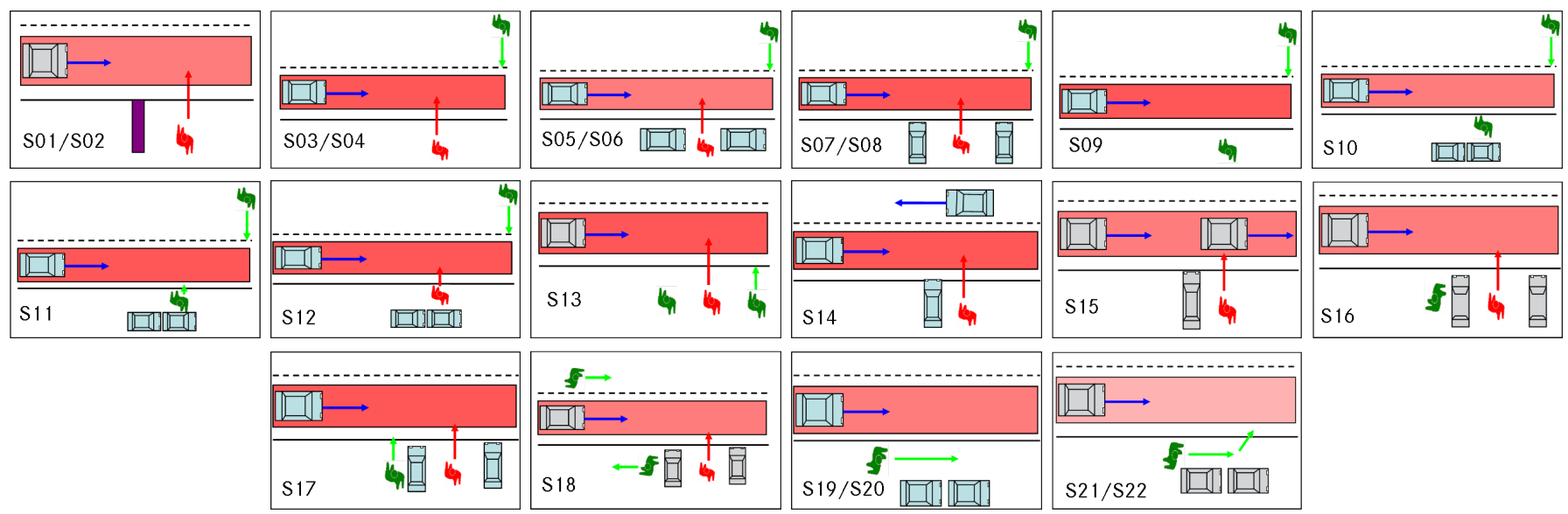

Fig. 6. Illustration of the 22 different scenarios, performed with real pedestrians (green) and a pedestrian dummy (red). Scenario pairs associated with a single diagram were performed with different dummy/pedestrian speeds, i.e. either $1 \mathrm{~m} / \mathrm{s}$ or $2 \mathrm{~m} / \mathrm{s}$.

\begin{tabular}{|l|c|c|c|c|}
\hline & 6D-Vision & PedRec (single-frame) & PedRecTrack & Fusion \\
\hline Sensitivity (frame level) & $66.2 \%(70.4 \%)$ & $75 \%$ & $76.2 \%$ & $88.9 \%$ \\
Precision (frame level) & N/A & N/A & $100 \%$ & $100 \%$ \\
\# False detected objects (frame level) & N/A & N/A & 0 & 0 \\
\hline Sensitivity (class-A trajectory) & $56.3 \%(62.8 \%)$ & $54.2 \%$ & $60.4 \%$ & $89.6 \%$ \\
Sensitivity (class-B trajectory) & $75.0 \%(83.7 \%)$ & $81.25 \%$ & $81.3 \%$ & $95.8 \%$ \\
Sensitivity (class-C trajectory) & $91.7 \%(100 \%)$ & $100 \%$ & $100 \%$ & $100 \%$ \\
\# False Trajectories & N/A & N/A & 0 & 0 \\
\hline Precision (class-A trajectory) & N/A & N/A & $100 \%$ & $100 \%$ \\
Precision (class-B trajectory) & N/A & N/A & $100 \%$ & $100 \%$ \\
Precision (class-C trajectory) & N/A & N/A & $100 \%$ & $100 \%$ \\
\hline
\end{tabular}

TABLE I

PEDESTRIAN DETECTION PERFoRMANCE OF BASELINE SySTEM (PEDRECTRACK, THIRD COLUMN) AND OF PROPOSED FUSION APPROACH (FUSION, LAST COLUMN) ON FULL DATASET, 22 SCENARIOS. BETWEEN BRACKETS, RESULTS ON DATA SUBSET CONTAINING MOVING PEDESTRIANS ONLY.

relying on motion, 6D-Vision cannot always be of help, however. Pedestrian standing or walking slowly (especially in longitudinal direction) are not well detected, which accounts for the somewhat lower detection rate (first column). As 6DVision is a generic moving object detection system, false pedestrian positives do not apply (see N/A entries).

Table II summarizes the obtained positional accuracy for the required pedestrians which were detected (i.e. within beforementioned localization tolerance). Lateral localization is quite accurate for all the 6D-Vision and PedRec components and fusion. Not surprisingly, longitudinal accuracy is lower for all variants. Here, PedRec has an edge, partly because its measurements are restricted to fully visible pedestrians.

\begin{tabular}{|l|c|c|c|}
\hline & 6D-Vision & PedRec (not tracked) & Fusion \\
\hline lateral & $0.06(0.06)$ & $0.05(0.05)$ & $0.06(0.05)$ \\
longitudinal & $0.40(0.16)$ & $0.17(0.17)$ & $0.32(0.31)$ \\
\hline
\end{tabular}

TABLE II

LOCALIZATION ACCURACY OVER DEFINED SENSOR COVERAGE AREA (LONGITUDINAL 7-27 M, LATERAL UP TO 6 M): ROOT MEAN SQUARED ERROR AND (BETWEEN BRACKETS) STANDARD DEVIATION IN METERS

For a reliable automatic vehicle maneuver, speed accuracy is important in addition to position accuracy. Figure 7 illustrates the estimated speed of the various configurations on scenario S01, from the time the pedestrian is partially visible coming behind the parked car. The speed of the pedestrian dummy $(2 \mathrm{~m} / \mathrm{s})$ is exactly known from the test setup.

Although the dummy is detected early by PedRecTrack system, the initial estimated position is not exact enough to allow a correct two-point filter initialization. This is because of small errors in depth estimation, caused by including disparity values belonging to the parked car that is occluding the dummy. Therefore, PedRecTrack is initialized with a speed of zero (same applies for the fused system). As Figure 7 shows, it takes about one second for the PedRecTrack system to converge to the correct speed of $2 \mathrm{~m} / \mathrm{s}$. The $6 \mathrm{D}$-Vision module, however, tracks the correct feature points on the moving target, and is able to converge fast to the correct speed. For the fused system, integrating the speed information from the 6D-Vision module helps the filter to converge faster to the correct speed than the baseline PedRecTrack system.

Finally, we analyze the performance regarding time-todetect, here defined as the number of frames it requires to detect a ground-truth trajectory, from first instance of full pedestrian visibility (a system trajectory that is started beyond the required sensor coverage range will result in a "time-todetect" of one frame). Trajectories that can not be detected by all configurations are excluded. A total of 42 trajectories 


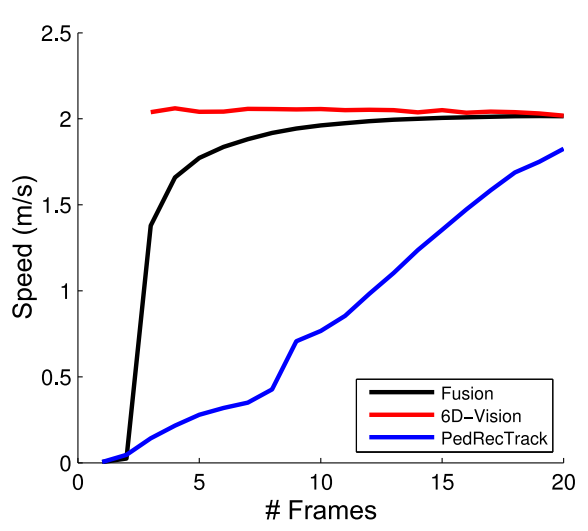

Fig. 7. Estimated pedestrian speed using the baseline PedRecTrack, 6DVision and the proposed fusion system. The ground truth speed is $2 \mathrm{~m} / \mathrm{s}$.

\begin{tabular}{|l|c|c|}
\hline PedRecTrack (baseline) & 6D-Vision & Fusion \\
\hline $2.4(2.8)$ & $1.4(1.3)$ & $1.5(1.6)$ \\
\hline
\end{tabular}

TABLE III

NUMBER OF FRAMES UNTIL THE PEDESTRIAN DUMMY IS DETECTED, FROM MOMENT OF FULL VISIBILITY: MEAN AND STANDARD DEVIATION (IN BRACKETS). DATA COMPUTED OVER 10 TRAJECTORIES, WITH INITIAL PARTIAL OCCLUSION.

remain, the results are shown in Figure 8. In analyzing the results of the individual sequences, we observe that lateral moving pedestrians $(2 \mathrm{~m} / \mathrm{s})$, for which the lower body part is occluded by the parked cars, are detected early by the 6D-Vision module, see Figure 9. Table III summarizes the results for this scenario subset. On the other hand, longitudinal moving pedestrians close to parked cars are more difficult to segment but pose no problem for the PedRec module. By fusing detections of both modules, the time to detect a pedestrian is reduced on average.

\section{Test of Integrated System}

We tested the integrated system (sensing, situation analysis, decision making and vehicle control) on two scenarios S01 and S02. In both scenarios, the vehicle drives close to $50 \mathrm{~km} / \mathrm{h}$ and the pedestrian dummy moves from the right side onto the vehicle's lane with a lateral speed of $2 \mathrm{~m} / \mathrm{s}$. In scenario S01 the pedestrian dummy is only partially occluded by a parking passenger vehicle. In scenario S02, the dummy appears behind a parking van and thus can only be detected by our system significantly later than in scenario S01. The desired vehicle action is to brake if still possible, otherwise to evade. See Figure 13 for snapshots of the integrated system choosing the correct vehicle action in scenarios S01 and S02.

We experimentally determined the last possible brake time for the vehicle to come to a complete stop to correspond to a pedestrian distance of $20 \mathrm{~m}$ (taking into account various device latencies). In scenario S01, the setup is such that the pedestrian is first fully visible at about $24 \mathrm{~m}$ distance ( $3.8 \mathrm{~m}$ lateral) to the vehicle. This means that the system has only about seven frames (corresponding to $4.1 \mathrm{~m}$ driven) to determine pedestrian position and speed, perform situation analysis and make the correct decision to initiate braking.

In scenario S02, the pedestrian dummy was initially occluded by a parking van aside of the road. Thus, the pedestrian dummy was only detected at a distance of less than $20 \mathrm{~m}$ and collision avoidance by braking was no longer possible. In the following example, the ego vehicle was driving with a constant speed of $45 \mathrm{~km} / \mathrm{h}$ and the pedestrian was first detected at a distance of $15.9 \mathrm{~m}$ and a lateral offset of $-3.4 \mathrm{~m}$. Figure 10 depicts the time-to-collision (TTC), time-to-brake (TTB), and time-to-steer (TTS) values provided by the situation analysis module of Section IV. As the pedestrian dummy becomes visible very late in this scenario, automatic braking can no longer avoid a collision and TTB $=-\infty$ throughout this measurement. As soon as TTS falls below a predefined total reaction time of the system (200 ms in our prototype system), an automatic steering maneuver is triggered and the TTX computation is stopped.
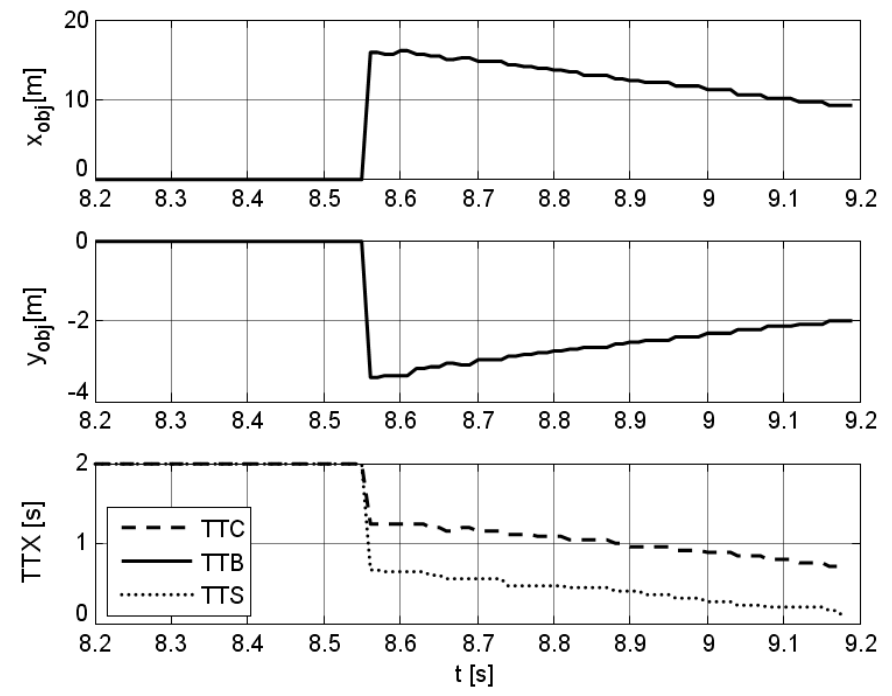

Fig. 10. Position of detected pedestrian (top, middle) and corresponding timeto-X values (bottom). Note that time-to-brake (TTB) is $-\infty$ in this sequence and thus not visible. An evasion maneuver is triggered at $t=9.18 \mathrm{~s}$.

Figure 11 shows the commanded trajectory $y_{t r j}$ and the reconstructed lateral position $y$ of the vehicle after the lateral controller has been started. Following Eq. (14), the actual lateral position $y$ was reconstructed using speed measurements and lateral acceleration measurements from odometry and inertial senors in our experimental vehicle. In this experiment, a fixed target lateral offset of $1 \mathrm{~m}$ has been chosen. As can be seen from the measurement data, the time lag between actual and commanded trajectory position is approx. $200 \mathrm{~ms}$. This time lag corresponds to the total reaction time of our system and is induced by our vehicle's dynamics, data processing time and the phase lag of the steering actuator.

Figure 12 show the measured lateral acceleration and vehicle speed during the automatic evasive maneuver. The maximum measured lateral acceleration is less than $10 \%$ higher than predefined limit of $a_{y, \max }=5 \mathrm{~m} / \mathrm{s}^{2}$, according to Eq. (7). This performance is acceptable in our application. The absolute speed of the vehicle is reduced by $3 \mathrm{~km} / \mathrm{h}$ during the maneuver.

We tested the integrated system by means of 20 runs on both scenarios S01 and S02. In all 40 runs, the prototype vehicle selected the correct action in time, not hitting the pedestrian dummy once. In the braking scenarios, the vehicle stopped 

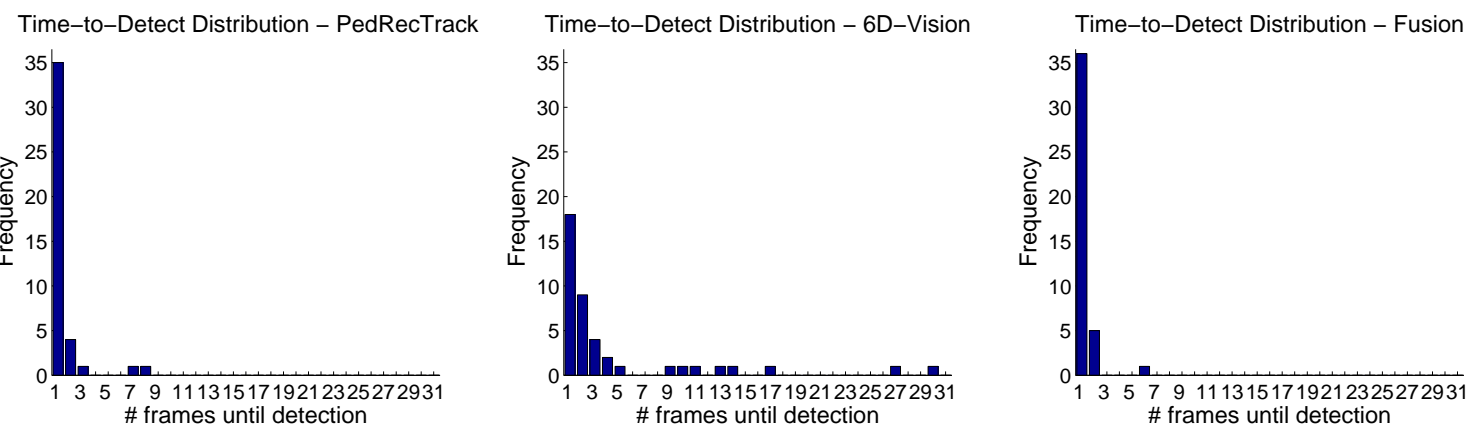

Fig. 8. Distribution of the number of frames until a pedestrian is detected, from the first frame of full visibility, for PedRecTrack, 6D-Vision and Fusion, respectively. Distribution over occluded and non-occluded trajectories that were detected (42 in total).

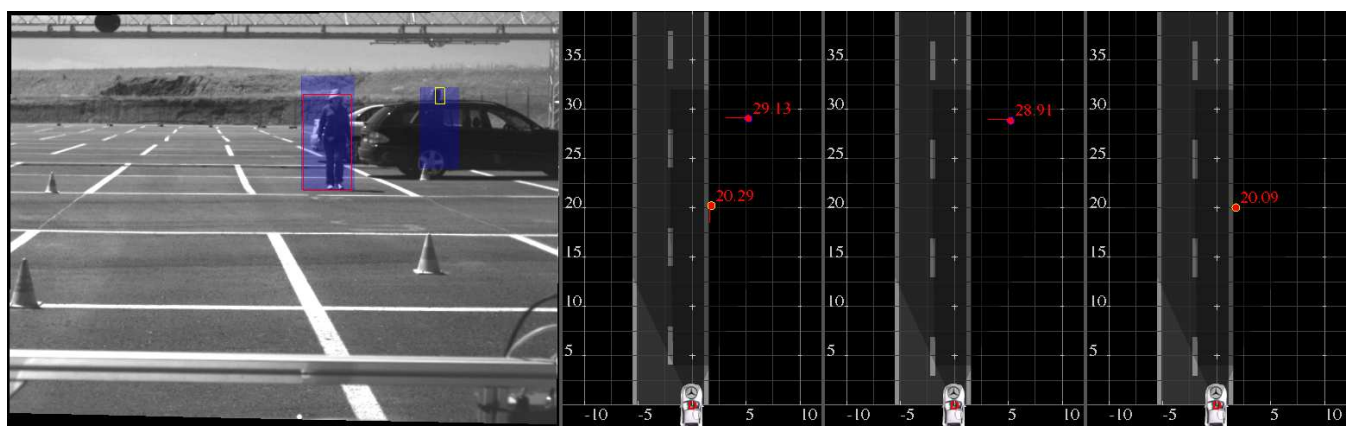

Fig. 9. An illustration of the complementary nature of PedRec with 6D-Vision. The grayscale image on the left displays the raw pedestrian detections (red box), 6D-Vision detections (small yellow box) and fusion results (blue box). The static fully visible pedestrian is detected by PedRec, the strongly occluded moving pedestrian is detected by $6 \mathrm{D}$-Vision. Both are detected by the fusion approach. To the right of the grayscale image, three top views associated with Fusion, 6D-Vision and PedRec. Numbers denote distance to vehicle.
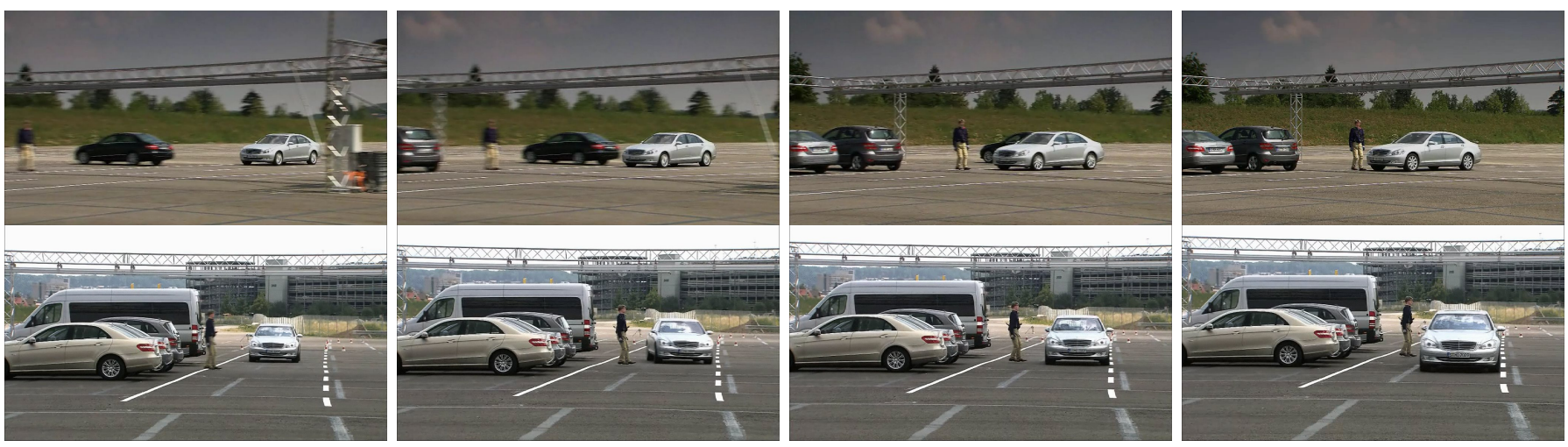

Fig. 13. (top) Braking scenario S01 vs. (bottom) Evasion scenario S02

approximately $30-150 \mathrm{~cm}$ ahead of the dummy.

\section{Discussion}

The previous section demonstrated a remarkably reliable vehicle system on the test track, that can detect pedestrians and make the right decision to brake or to evade, in a split of a second. There are a number of technical challenges associated with extending the flawless performance of the system on the test track to the real urban traffic environment,

Regarding the sensing component, note that for our experimental setting on the test track, it was easy to discard 6D-Vision detections on moving vehicles, based on speed considerations. Therefore, the remaining 6D-Vision detections, associated with realistic pedestrian speeds, were treated similarly to the PedRec detections in the fusion approach of Section III-C. The decision whether to output a track was solely based on the number of detections, irrespective of their source. In a real traffic environment, there will be many other moving objects, which could be pedestrian-like. Future work will develop a probabilistic approach, which maps 6DVision and PedRec detections onto posteriors for pedestrians, taking into account bounding box sizes, locations, speeds and classifier decision values. The decision whether to initiate a track would be made by analyzing the cumulative probability of observing a pedestrian.

It is paramount to avoid false system activations (i.e. unnecessary braking or evasion maneuver). For that, all system modules and in particular the sensing component (6DVision, PedRec) will need to be enhanced (e.g. better position and velocity estimation, recognition performance, recognizing pedestrians under partial occlusion [17]). Sensor fusion (e.g. with radar, laser scanners) can provide an additional level of 

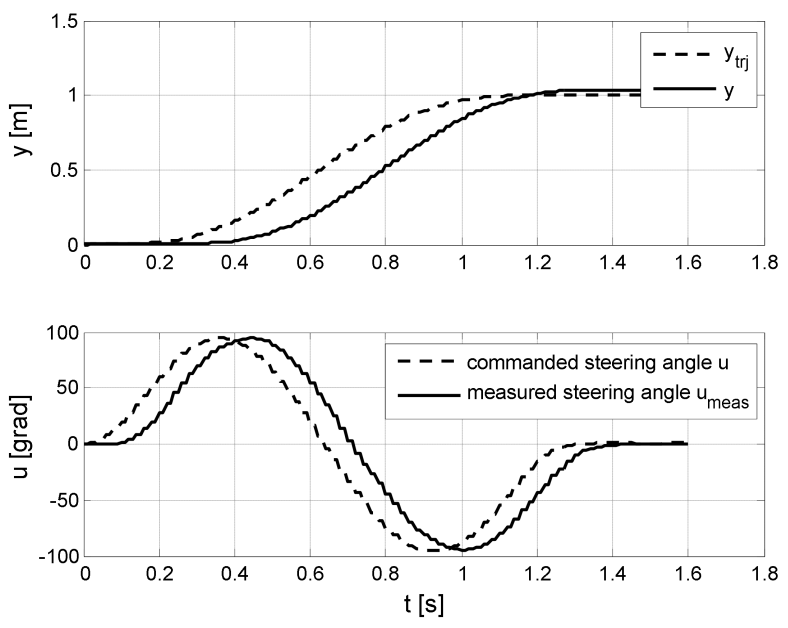

Fig. 11. Commanded trajectory and measurement results after evasion has been triggered. Top: Lateral position of the vehicle. Bottom: Steering wheel angle. The upper plot shows a total reaction time of the vehicle's of approx. $200 \mathrm{~ms}$; this includes a steering actuator phase lag of about $70 \mathrm{~ms}$ as depicted in the lower diagram.

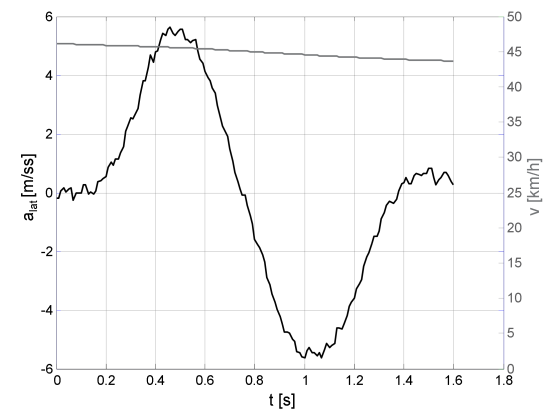

Fig. 12. Measured lateral acceleration and vehicle speed.

robustness.

The sensing component might be extended to other traffic participants, such as bicylists and cars, to match the capabilities of the current situation analysis component. The current evasion maneuver results in a lateral offset of $80-100 \mathrm{~cm}$ of the vehicle. Larger offsets are conceivable. This places demands that the sensing component also performs a free space analysis [44], to verify that the automatic evasion maneuvre can indeed be safely performed. Being able to detect elements of the traffic infrastructure (e.g. lane markings, traffic lights) will furthermore enable more sophisticated situation analysis.

As a final note, we emphasize that the presented system is meant for emergency situations, in which the driver will likely not be in a position to still act properly. Vehicle control (and responsibility) rests, however, fully with the driver; at each time instant the driver can overrule the system, by either accelerating or maintaining a grip on the steering wheel.

\section{CONCLUSION}

This paper presented a novel active pedestrian safety system, which combines sensing, situation analysis, decision making and vehicle control. The vision sensing component fuses two complementary approaches: generic motion-based object detection (6D-Vision) and pedestrian recognition (PedRec). Situation analysis was based on numerical simulation, which allowed to incorporate more complex, non-circular vehicle paths based on a polynomial model. Decision making involved the continuous monitoring of time-to-collision, time-to-brake and time-to-steer measures, and initiating a specialized control loop in case of an evasion maneuver.

We performed extensive pre-crash experiments with the system on the test track. We demonstrated that the benefit of adding 6D-Vision to a baseline PedRec(Track) system is that lateral moving pedestrians $(2 \mathrm{~m} / \mathrm{s})$ can be detected earlier when partially occluded by a parked car, and furthermore, velocity estimation is more accurate. On two scenarios, requiring a split-second decision between no action, automatic braking and automatic evasion, the system made in all runs (over 40) the correct decision. Despite the strong performance on the test track, additional challenges remain before this system can reliably be deployed in real urban traffic.

\section{REFERENCES}

[1] IRTAD, "International traffic safety data and analysis group," in http://www.internationaltransportforum.org/home.html, 2006.

[2] T. Gandhi and M. Trivedi, "Pedestrian protection systems: Issues, survey, and challenges," IEEE Trans. on ITS, vol. 8, no. 3, pp. 413-430, 2007.

[3] M. Enzweiler and D. M. Gavrila, "Monocular pedestrian detection: Survey and experiments," IEEE Trans. on PAMI, vol. 31, no. 12, pp. 2179-2195, 2009.

[4] P. Viola, M. Jones, and D. Snow, "Detecting pedestrians using patterns of motion and appearance," IJCV, vol. 63, no. 2, pp. 153 - 161, 2005.

[5] Q. Zhu, M. Yeh, K. Chen, and S. Avidan, "Fast human detection using a cascade of histograms of oriented gradients," in Proc. of the IEEE CVPR, vol. 2, 2006, pp. 1491-1498.

[6] I. Alonso et al., "Combination of feature extraction methods for SVM pedestrian detection," IEEE Trans. on ITS, vol. 8, no. 2, pp. 292-307, 2007.

[7] A. Broggi, A. Fascioli, I. Fedriga, A. Tibaldi, and M. D. Rose, "Stereobased preprocessing for human shape localization in unstructured environments," in Proc. of the IEEE IV, 2003, pp. 410-415.

[8] A. Ess, B. Leibe, and L. van Gool, "Depth and appearance for mobile scene analysis," in Proc. of the ICCV, 2007.

[9] D. M. Gavrila and S. Munder, "Multi-cue pedestrian detection and tracking from a moving vehicle," IJCV, vol. 73, no. 1, pp. 41-59, 2007.

[10] S. Munder, C. Schnörr, and D. M. Gavrila, "Pedestrian detection and tracking using a mixture of view-based shape-texture models," IEEE Trans. on ITS, vol. 9, no. 2, pp. 333-343, 2008.

[11] M. Enzweiler, P. Kanter, and D. Gavrila, "Monocular pedestrian recognition using motion parallax," in Proc. of the IEEE IV, 2008, pp. $792-$ 797.

[12] D. M. Gavrila, "A Bayesian, exemplar-based approach to hierarchical shape matching," IEEE Trans. on PAMI, vol. 29, no. 2, pp. 1408-1421, 2007.

[13] M. Enzweiler and D. Gavrila, "A mixed generative-discriminative framework for pedestrian classification," in Proc. of the IEEE CVPR, 2008.

[14] C. Papageorgiou and T. Poggio, "A trainable system for object detection," IJCV, vol. 38, pp. 15-33, 2000.

[15] N. Dalal and B. Triggs, "Histograms of oriented gradients for human detection," in Proc. of the IEEE CVPR, vol. 1, 2005, pp. 886-893.

[16] L. Oliveira, U. Nunes, and P. Peixoto, "On exploration of classifier ensemble synergism in pedestrian detection," IEEE Trans. on ITS, vol. 11, no. 1, pp. 16-27, 2010.

[17] M. Enzweiler, A. Eigenstetter, B. Schiele, and D. Gavrila, "Multi-cue pedestrian classification with partial occlusion handling," in Proc. of the IEEE CVPR, 2010.

[18] M. Bajracharya et al., "A fast stereo-based system for detecting and tracking pedestrians from a moving vehicle," Int. J. Rob. Research, vol. 28, 2009.

[19] A. Broggi et al., "Scenario-driven search for pedestrians aimed at triggering non-reversible systems," in Proc. of the IEEE IV, 2009, pp. 285-291.

[20] K. Fuerstenberg, "Pedestrian protection based on laserscanners," in Proc. of the ITS, 2005.

[21] D. Llorca et al., "An experimental study on pitch compensation in pedestrian-protection systems for collision avoidance and mitigation," IEEE Trans. on ITS, vol. 10, no. 3, pp. 469-474, 2009. 
[22] P. Marchal, M. Dehesa, D. M. Gavrila, M. Meinecke, N. Skellern, and V. Vinciguerra, "Final report," Deliverable 27, EU Project SAVE-U, 2005.

[23] M. Meinecke, M. Obojski, M. Töns, and M. Dehesa, "SAVE-U: First experiences with a pre-crash system for enhancing pedestrian safety," in Proc. of the ITS, 2005.

[24] S. Nedevschi, S. Bota, and C. Tomiuc, "Stereo-based pedestrian detection for collision-avoidance applications," IEEE Trans. on ITS, vol. 10, no. 3, pp. 380-391, 2009.

[25] H. Ju, B. Kwak, J. Shim, and P. Yoon, "Precrash dipping node (PCDN) needs pedestrian recognition," IEEE Trans. on ITS, vol. 9, no. 4, pp. $678-687,2008$.

[26] J. Hillenbrand, A. Spieker, and K. Kroschel, "A multilevel collision mitigation approach," IEEE Trans. on ITS, vol. 7, no. 4, pp. $528-540$, 2006.

[27] S. Lavalle, "Rapidly-exploring random trees: A new tool for path planning," Computer Science Dept, Iowa State University, Tech. Rep. 9811, 1998.

[28] P. Fiorini and Z. Shiller, "Time optimal trajectory planning in dynamic environments," Proc. of the ICRA, vol. 2, pp. 1553-1558, 1996.

[29] C. Schmidt, F. Oechsle, and W. Branz, "Research on trajectory planning in emergency situations with multiple objects," in Proc. of the IEEE ITSC, 2006, pp. 988 - 992.

[30] Y. Hattori, E. Ono, and S. Hosoe, "Optimum vehicle trajectory control for obstacle avoidance problem," IEEE/ASME Trans. on Mechatronics, vol. 11, pp. $507-512,2006$.

[31] R. Isermann, M. Schorn, and U. Stählin, "Anticollision system proreta with automatic braking and steering," Vehicle System Dynamics, vol. 46, pp. $683-694,2008$.

[32] DARPA, "Urban challenge," in http://www.darpa.mil/grandchallenge/, 2007.

[33] U. Franke and A. Joos, "Real-time stereo vision for urban traffic scene understanding," in Proc. of the IEEE IV, October 2000, pp. 273-278.

[34] H. Hirschmueller, "Accurate and efficient stereo processing by semiglobal matching and mutual information," in Proc. of the IEEE CVPR, vol. 2, June 2005, pp. 807-814.

[35] M. Martin and H. Moravec, "Robot evidence grids," Tech. Rep. CMURI-TR-96-06, 1996.

[36] U. Franke, C. Rabe, H. Badino, and S. Gehrig, "6d-vision: Fusion of stereo and motion for robust environment perception," in Proc. of the DAGM, 2005, pp. 216-223.

[37] C. Rabe, U. Franke, and S. Gehrig, "Fast detection of moving objects in complex scenarios," in Proc. of the IEEE IV, 2007, pp. 398-403.

[38] H. Badino, "A robust approach for ego-motion estimation using a mobile stereo platform," in 1st Int. Workshop on Complex Motion (IWCM04), Guenzburg, Germany, October 2004.

[39] J. Klappstein, F. Stein, and U. Franke, "Monocular motion detection using spatial constraints in a unified manner," in Proc. of the IEEE IV, 2006, pp. 261-267.

[40] C. Tomasi and T. Kanade, "Detection and tracking of point features," Tech. Rep. CMU-CS-91-132, 1991.

[41] U. Kiencke and L. Nielsen, Automotive Control Systems: For Engine, Driveline and Vehicle. Springer-Verlag, 2000.

[42] H. Fritz, "Vorrichtung zur Durchführung eines Fahrspurwechsels." German Patent DE 10012737 B4, 2001.

[43] _ _ "Verfahren und Vorrichtung zur Kollisionsvermeidung für ein Fahrzeug durch Ausweichen vor einem Hindernis. German Patent Disclosure DE 102009020648 A1," German Patent, 2009.

[44] H. Badino, R. Mester, T. Vaudrey, and U. Franke, "Stereo-based free space computation in complex traffic scenarios," in SSIAI, Santa Fe, NM, Mar. 2008, pp. 189-192.

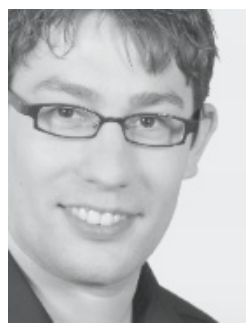

Christoph G. Keller received the MSc degree in computer science from the Univ. of Freiburg, Germany in 2007. In 2006 and 2007 he was a visiting student researcher at Siemens Corporate Research in Princeton, USA. Since 2007, he has been a PhD student with the Image \& Pattern Analysis Group at the Univ. of Heidelberg, Germany, while on site at Daimler R\&D in Ulm, Germany. His current research focuses on pedestrian detection and tracking in the context of intelligent vehicles.

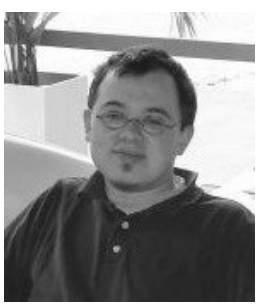

Thao Dang (M04) studied electrical engineering at the Techn. Univ. of Karlsruhe, Germany, the MIT, Cambridge, and the Univ. of Massachusetts, Dartmouth. He received the Dr.-Ing. degree $(\mathrm{PhD})$ with distinction from the Univ. of Karlsruhe in 2007. Since September 2007, he has been a research engineer at Daimler AG, Germany. His research interests include stereo vision, camera self-calibration, and driver assistance systems. Dr. Dang received the IEEE ITSS Best Dissertation Award in 2007 and the Measurement Technology Award 2008 of the Arbeitskreis der Hochschullehrer für Messtechnik (AHMT).

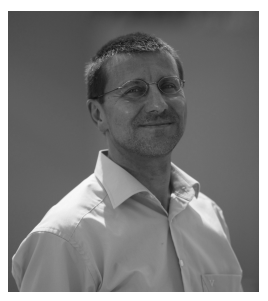

Hans Fritz obtained his Dipl.-Ing. degree in electrical engineering from the Techn. Univ. of Karlsruhe in 1985. From 1985 to 1986 he was research assistant at the Inst. of System Dynamics and Control at the Univ. of Stuttgart. Since 1986 he is with Daimler R\&D in Stuttgart, Germany. He received his Dr.-Ing. degree in control engineering from the Univ. of Stuttgart in 1997. His main area of research is advanced driver assistance systems, particularly longitudinal and lateral control of road vehicles.

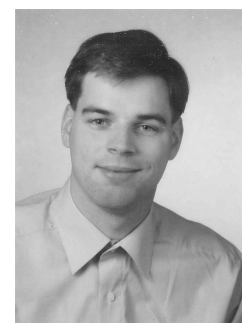

Armin Joos Armin Joos received the degree of mathematics from the university of applied sciences of Stuttgart in 1999. Afterwards he became a senior research scientist at Daimler Research in Esslingen and Böblingen, Germany. He has started his research on stereo vision in the departement for computer vision. Since 2001 he has focused his work on driver assistance systems for collision mitigation and avoidance.

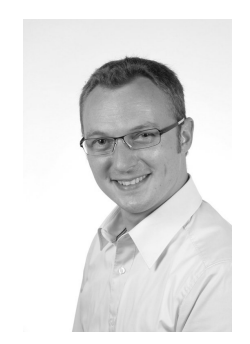

Clemens Rabe was born 1979 in Giessen, Germany. $\mathrm{He}$ received his diploma degree in computer engineering from the University of Applied Sciences Wuerzburg, Germany in 2005 . He is currently pursuing a Ph.D. degree in computer science at the University of Kiel in cooperation with the Environment Perception Group at Daimler Research, Sindelfingen. His research interests are in stereo vision, motion analysis, and traffic scene understanding.

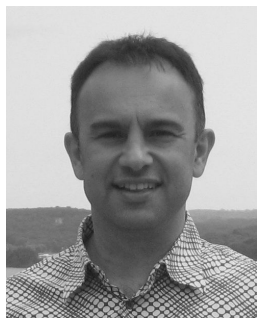

Dariu M. Gavrila received the $\mathrm{PhD}$ degree in comp. sc. from the Univ. of Maryland at College Park in 1996. He was a visiting researcher at the MIT Media Lab in 1996. Since 1997, he has been a senior research scientist at Daimler R\&D in Ulm, Germany. In 2003, he was named professor at the Univ. of Amsterdam, in the area of intelligent perception systems (part time). Prof. Gavrila focuses on visual systems for detecting human presence and activity, with application to intelligent vehicles and surveillance. He received the I/O Award 2007 from the Dutch Science Foundation (NWO). His personal Web site is www.gavrila.net. 